\title{
Evaluation of the Lens-absorbed Dose of the Scattered Radiation Generated During Tomotherapy IMRT to the H\&N Cancer Patient
}

\author{
Jae-Won Choi ${ }^{1}$, Hae-Kag Lee ${ }^{2}$, Jae-Hwan Cho ${ }^{3}$, Cheon Woong Choi ${ }^{4}$, \\ Myung Sik Ju' ${ }^{5}$, Bok Soon Chang ${ }^{6}$, and Cheol-Soo Park ${ }^{7 *}$ \\ ${ }^{1}$ Department of Radiation Oncology, Samsung Medical Center, Seoul Iron 06351, Korea \\ ${ }^{2}$ Department of Computer Science and Engineering, Soonchunhyang University, Asan 31538, Korea \\ ${ }^{3}$ Department of Radiological Technology, Ansan University, Ansan 15518, Korea \\ ${ }^{4}$ Department of Respiratory and Critical Care Medicine, Kyung Hee University Hospital at Gangdong, Seoul 05278, Korea \\ ${ }^{5}$ Department of Radiology, Soonchunhyang University Bucheon Hospital, 14584, Korea \\ ${ }^{6}$ Department of Pulmonary and Critical Care Medicine, Kyung Hee Univeristy Hospital at Gangdong, School of Medicine, \\ Kyung Hee University, Seoul 05278, Korea \\ ${ }^{7}$ Department of Radiological Science, Hallym Polytechnic University, Chuncheon 24210, Korea
}

(Received 12 January 2017, Received in final form 6 February 2017, Accepted 6 February 2017)

\begin{abstract}
This paper uses a glass dosimeter to evaluate the lens-absorbed dose of scattered radiation generated in tomotherapy intensity modulated radiation therapy (IMRT). The head and neck portion of the rando phantom was subjected to a CT scan. The tomotherapy plan was designed to ensure delivery of the prescribed total 70 Gy day 2.2 Gy. With the lens portion of the glass dosimeter, a $5 \mathrm{~mm}$ bolus was subjected to the scattered radiation treatment, and the dose was measured in each of the three megavoltage CT (MVCT) modes. The result is multiplied by 30 times and was determined once as the mean value. The measurement at the MVCT Coarse mode is RT mode $10.797 \mathrm{mGy}$, that for the Normal mode is $13.360 \mathrm{mGy}$, for the Fine mode is a maximum of $22.872 \mathrm{mGy}$, and for the treatment mode is $895.830 \mathrm{mGy}$. A small amount of scattered radiation in the MVCT is measured in the lens scattered radiation, but scattered radiation during treatment was measured to be near $1 \mathrm{~Gy}$ on the lens. Compared to a one-time radiation treatment of $2.2 \mathrm{~Gy}$, the survey showed something unexpected in that it was half the value of that research to the patient. Therefore, will be aware of how much of an influence there will be on sensitive organs, such as the lens by scattered radiation generated during intensity modulated radiation therapy.
\end{abstract}

Keywords : scattered radiation, IMRT (intensity modulated radiation therapy), absorbed dose, tomotherapy, MVCT (megavoltage CT)

\section{Introduction}

Treatment for head and neck cancer (H \& N Cancer) involves a combination of radiotherapy and chemotherapy. Radiation therapy has been developed into intensity modulated radiation therapy (IMRT), which is performed through conventional 2D radiotherapy and 3D stereotactic radiotherapy [1]. The head and neck neck are complicated, with various organs, and a pencil beam can be designed to control the intensity of the radiation therapy in various organs and to achieve local control of the initial head and

CThe Korean Magnetics Society. All rights reserved.

*Corresponding author: Tel: +82-33-240-9350

Fax: +82-33-240-9354, e-mail: pcs3109@hsc.ac.kr neck cancers $(\mathrm{T} 1, \mathrm{~T} 2)(\mathrm{T} 3, \mathrm{~T} 4) .44-73 \%$ of patients with advanced head and neck cancer (87-100 \%) showed better response to other types of site radiotherapy, and the patient was easily fixed using an aquaplast mask (WFRAquaplast/Qfix System, Avondale, pennsylvania) that makes treatment possible [2]. However, the scattering line generated during the megavoltage CT (MVCT) scan and the radiotherapy process are anticipated to expose radiationsensitive organs. When an absorbed dose other than the computerized treatment plan is given to a patient, critical organs including the spinal cord, brainstem, optic nerve, optic disc, parotid gland, and lens can be included, depending on the size and location of the tumor. Among various organs, the lens is an important structure that is located in the anterior part of the eye, and it is transparent 
to the iris behind it and collects light. The lens is $4 \mathrm{~mm}$ in thickness, $9 \mathrm{~mm}$ in diameter, and is convex on both sides. It is sensitive to radiation, so a careful observation of absorbed doses should minimize the risk of secondary radiation injury, such as cataract or lens opacity, to ensure maximum quality of life after treatment [3]. Recent studies have shown that the lens located near the surface of the body is at risk of cataracts at $4 \mathrm{~Gy}$ and that lens opacity that may occur at 0.5-2 Gy, with a reduction in the threshold dose as radiation therapy proceeds [4]. In addition, caution should be exercised when acquiring images for imaging-guided radiation therapy because $5 \%$ of 5-year-long adverse effects may occur when $10 \mathrm{~Gy}$ is exceeded for the lens during radiotherapy [5].

\section{Subjects and Method}

\subsection{CT simulation}

The aquaplast mask was prepared using a phantom (Anderson rando phantom, USA), and a $2.5 \mathrm{~mm}$ slice thickness CT scan was performed to cover the neck to the head (LightSpeed RT 16, General Electric, USA).

\subsection{Treatment RT Planning}

Contouring the patient's tumor location and size from the acquired image was planned in Phantom, so that no dose was absorbed by the lens in the treatment plan. The Clinical Target Volume (CTV) has a margin of $3 \mathrm{~mm}$ for the gross tumor volume (GTV), and the target volume for planning (PTV) is $95 \%$ for 70 Gy in CTV $+3 \mathrm{~mm}$ (Tomotherapy, Inc, USA) (Fig. 1, Radiation therapy). The daily doses and total number of doses may vary depending on the patient's progression stage and size. However, the baseline attempt was made at 18 Gy per day at 2.2
Gy, and 12 doses added to 2.4 Gy. However, since this study focuses on scattering lines that can occur in intensity modulated radiotherapy, treatment is assumed to be performed at $2.2 \mathrm{~Gy}$ for the same 10 times.

\subsection{Measurement method of absorbed dose of cata-} ract

To accurately measure the radiation, the glass dosimeter was calibrated by irradiating $6 \mathrm{mGy}$ to the glass element using standard source $137 \mathrm{Cs}$. To remove the background value of the glass dosimeter (GD-352M, CHIYODA TECHNOL CORPORATION), the glass dosimeter was annealed at $400{ }^{\circ} \mathrm{C}$ for 2 hours. The measured device was measured by preheating at $80{ }^{\circ} \mathrm{C}$ for 1 hour before the reading the dose value. FDG-1000 (CHIYODA TECHNOL CORPORATION) was used as the reading instrument for the glass dosimeter.

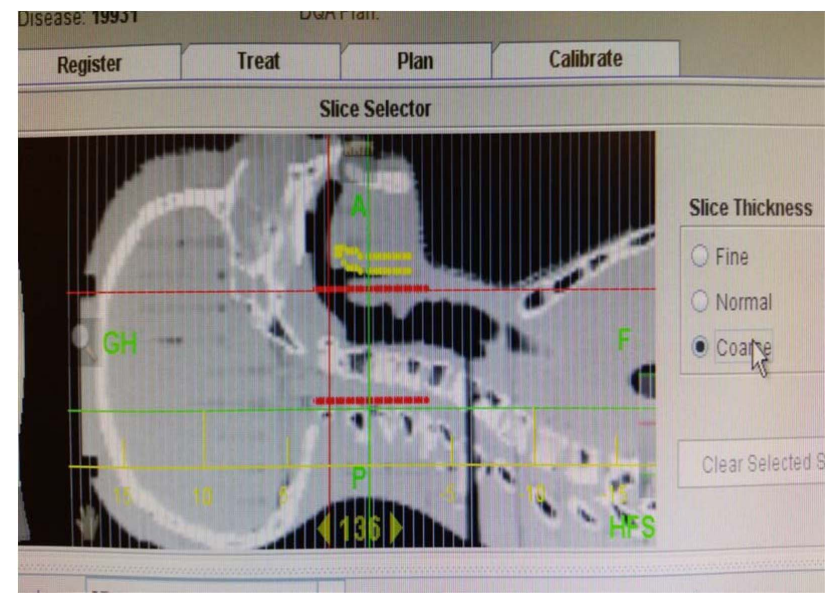

Fig. 2. (Color online) Mega Voltage Computed Tomography.

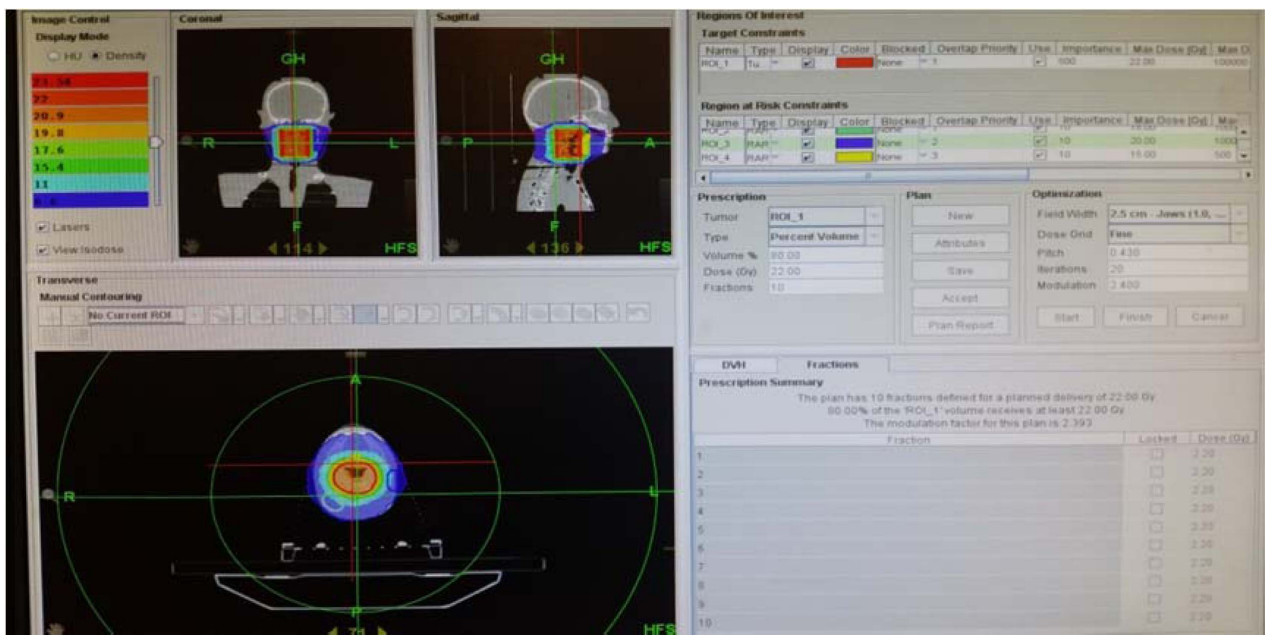

Fig. 1. (Color online) Treatment RT Planning. 
Table 1. Megavoltage CT Irradiation condition.

\begin{tabular}{ccccc}
\hline \hline $\begin{array}{c}\text { Exposure } \\
\text { conditions }\end{array}$ & $\begin{array}{c}\text { Exposure } \\
\text { thickness } \\
(\mathrm{mm})\end{array}$ & $\begin{array}{c}\text { Exposure } \\
\text { time } \\
(\mathrm{sec})\end{array}$ & Energy & $\begin{array}{c}\text { Jaw size } \\
(\mathrm{mm})\end{array}$ \\
\hline Fine & 2 & 206 & $3.5 \mathrm{MV}$ & 4 \\
Normal & 4 & 116 & $3.5 \mathrm{MV}$ & 4 \\
Coarse & 6 & 81 & $3.5 \mathrm{MV}$ & 4 \\
\hline
\end{tabular}

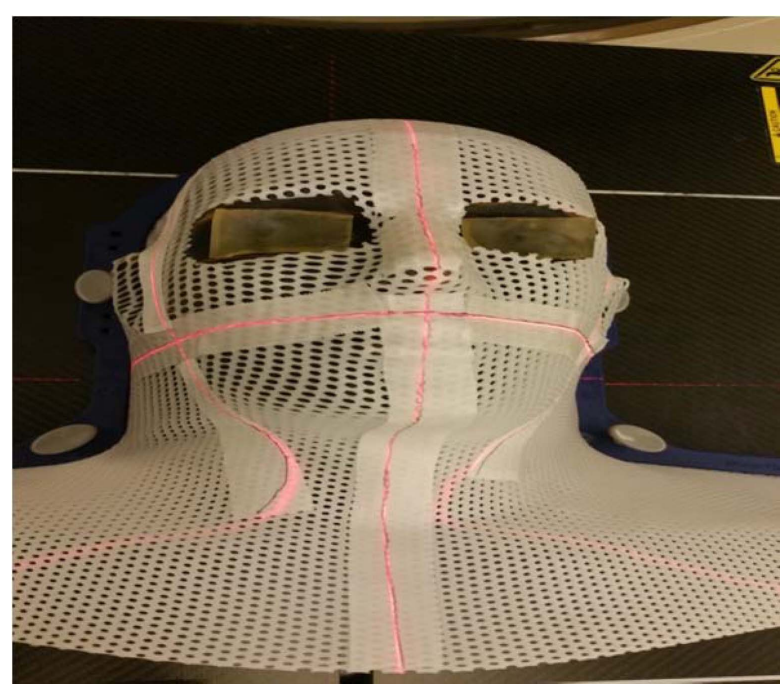

Fig. 3. (Color online) $5 \mathrm{~mm}$ Bolus apply.

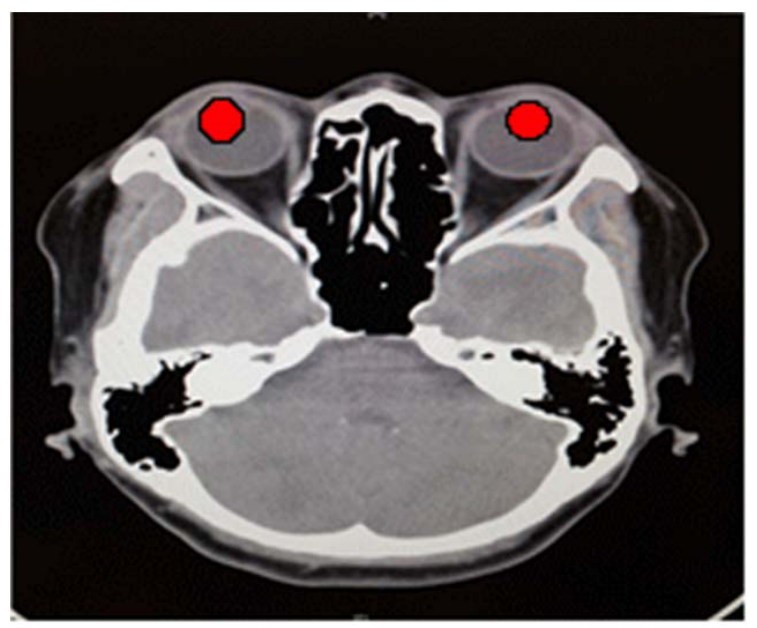

Fig. 4. (Color online) Location of glass dosimeter.

\subsection{Method of measuring dose}

Tomotherapy HD generally uses $6 \mathrm{MV}$ of single energy for treatment and 3.5 MV for the MVCT scan. The MVCT Scan has three modes - coarse, normal, and fine - which capture images as the couch moves during one rotation of the Gentry, and these usually consisting of $6 \mathrm{~mm}$ (coarse), $4 \mathrm{~mm}$ (normal), and $2 \mathrm{~mm}$ (fine) (Fig. 2) (Table 1). A 5$\mathrm{mm}$ bolus was placed on the left side of the phantom's eye and the anatomical structure of the lens. The measurement was repeated ten times in three modes with MVCT, 10 times, and the average value was calculated by summing all results (Figs. 3, 4).

\section{Result}

In the case of Tomotherapy MVCT, the measured value of the absorbed dose of the lens is $0.360 \mathrm{mGy}$ for coarse, $0.336 \mathrm{mGy}$ for LT, $0.446 \mathrm{mGy}$ for normal, $0.412 \mathrm{mGy}$ for LT, $0.762 \mathrm{mGy}$ for RT, and LT $729 \mathrm{mGy}$ for fine (Tables $2-4)$. The measured value is the average value measured ten times, and the difference between the average value of coarse and fine measurements is about two times at maximum. Since the total treatment plan is established more than 30 times to treat head and neck cancer, the

Table 2. Coarse megavoltage CT Crystal scattering ray absorption dose.

\begin{tabular}{ccc}
\hline \hline Number of & \multicolumn{2}{c}{ Coarse } \\
\cline { 2 - 3 } measurements & Rt lens & Lt lens \\
\hline 1st & 0.394 & 0.335 \\
2nd & 0.399 & 0.335 \\
3rd & 0.367 & 0.323 \\
4th & 0.368 & 0.324 \\
5th & 0.333 & 0.327 \\
6th & 0.329 & 0.328 \\
7th & 0.356 & 0.350 \\
8th & 0.359 & 0.350 \\
9th & 0.345 & 0.345 \\
10th & 0.349 & 0.346 \\
Mean & 0.360 & 0.336 \\
30 frac. & 10.797 & 10.089 \\
\hline
\end{tabular}

Table 3. Normal megavoltage CT lens scattered radiation absorbed dose. (Unit: $\mathrm{mGy}$ )

\begin{tabular}{ccc}
\hline \hline Number of & \multicolumn{2}{c}{ Normal } \\
\cline { 2 - 3 } measurements & Rt lens & Lt lens \\
\hline 1st & 0.492 & 0.438 \\
2nd & 0.487 & 0.433 \\
3rd & 0.431 & 0.434 \\
4th & 0.429 & 0.435 \\
5th & 0.446 & 0.427 \\
6th & 0.445 & 0.424 \\
7th & 0.401 & 0.368 \\
8th & 0.402 & 0.370 \\
9th & 0.462 & 0.395 \\
10th & 0.461 & 0.396 \\
Mean & 0.446 & 0.412 \\
30 frac. & 13.368 & 12.360 \\
\hline
\end{tabular}


Table 4. Fine megavoltage CT lens scattered radiation absorbed dose.

\begin{tabular}{ccc}
\hline \hline \multirow{2}{*}{$\begin{array}{c}\text { Number of } \\
\text { measurements }\end{array}$} & \multicolumn{2}{c}{ Fine } \\
\cline { 2 - 3 } & Rt lens & Lt lens \\
\hline 1st & 0.786 & 0.728 \\
2nd & 0.778 & 0.734 \\
3rd & 0.765 & 0.740 \\
4th & 0.677 & 0.702 \\
5th & 0.680 & 0.699 \\
6th & 0.833 & 0.764 \\
7th & 0.829 & 0.767 \\
8th & 0.748 & 0.718 \\
9th & 0.749 & 0.721 \\
10th & 0.779 & 0.723 \\
Mean & 0.762 .4 & 0.730 \\
30 frac. & 22.872 & 21.888 \\
\hline
\end{tabular}

Table 5. Tomotherapy Lens scattered radiation absorbed dose.

\begin{tabular}{ccc} 
& \multicolumn{2}{c}{ (Unit: mGy) } \\
\hline \hline \multirow{2}{*}{$\begin{array}{c}\text { Number of } \\
\text { measurements }\end{array}$} & Rt lens & Lt lens \\
\cline { 2 - 3 } & 30.710 & 28.620 \\
1st & 30.780 & 28.600 \\
2nd & 27.910 & 29.550 \\
3rd & 30.940 & 31.960 \\
4th & 30.900 & 31.940 \\
5th & 30.160 & 26.390 \\
6th & 31.550 & 26.370 \\
7th & 27.490 & 26.400 \\
8th & 27.440 & 26.380 \\
9th & 30.730 & 28.580 \\
10th & 29.861 & 28.479 \\
Mean & 895.830 & 854.370 \\
30 frac. & &
\end{tabular}

absorbed dose generated by acquiring the pre-treatment image is 30 times the average measurement value, and the maximum is RT $22.872 \mathrm{mGy}$, LT $21.888 \mathrm{mGy}$, and the minimum is RT $10.797 \mathrm{mGy}$, LT 10.089 mGy (Table 2) (Fig. 5). Intensity modulated radiotherapy was used to measure the amount of absorbed dose of the lens at RT $29.296 \mathrm{mGy}$, and LT $28.479 \mathrm{mGy}$, respectively. This measurement is the average value measured ten times. In addition, since the total treatment was applied more than 30 times, the amount of absorbed dose of the lens produced during treatment is 308 times the average value, resulting in RT $895.83 \mathrm{mGy}$ and LT $854.37 \mathrm{mGy}$. Assuming 30 times of MVCT and tomotherapy radiation treatment, the maximum dose of the lens was about 40 times higher than that of $22.872 \mathrm{mGy}$ at $895.830 \mathrm{mGy}$ (Table 5) (Fig. 5).

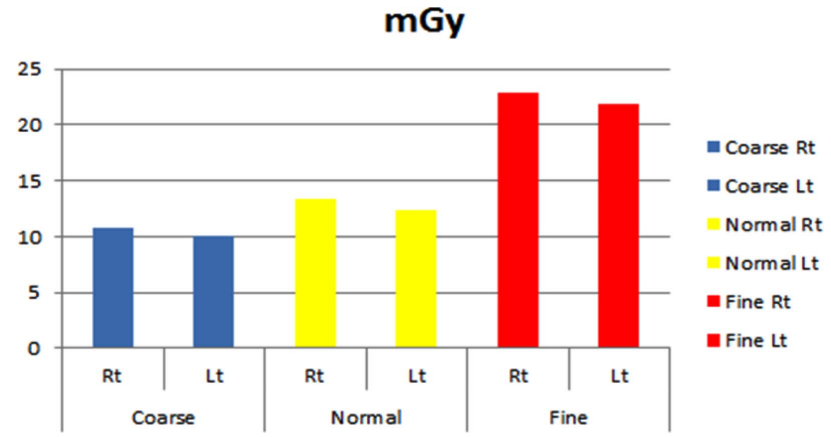

Fig. 5. (Color online) Compared to the amount of absorbed dose of lens by MVCT irradiation condition.

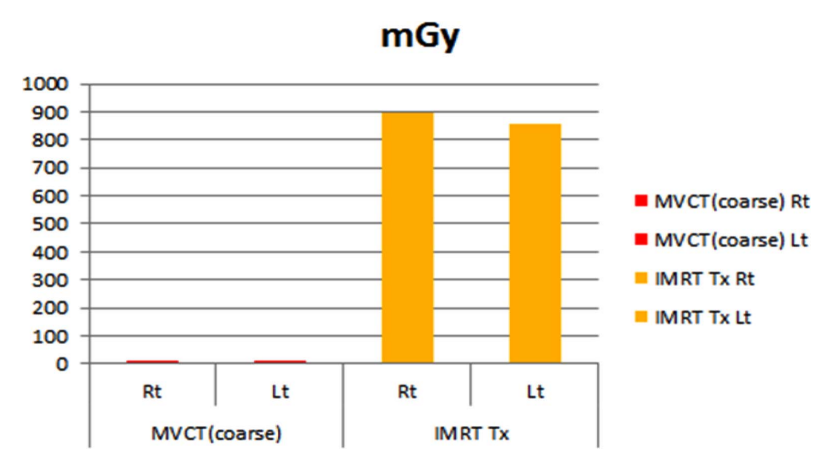

Fig. 6. (Color online) Comparison of MVCT and Intensity Modulated Radiation Therapy Lens Scattering Absorption Dose.

\section{Discussion}

In the case of head and neck cancer, if the tumor is located close to the lens or there is overlap in the MVCT or radiotherapy, the data in this paper show a measured value that cannot be ignored if the lens's constant is set near the limit dose. In the radiation therapy oncology group (RTOG) 0539, a total of 60 Gy or more was applied to the planning target volume (PTV) in the IMRT treatment and a total of 30 Gy [6-10]. We recommend that the maximum limit of the absorbed dose of the lens be $7 \mathrm{~Gy}$. In a clinical setting, the limited dose of the lens is often set to 5-6 Gy. If an unexpected dose of 1-2 Gy is absorbed by the lens in such a situation, the scattering line should be carefully considered because it exceeds the recommended $7 \mathrm{~Gy}$ and may interfere with the lens. The reason to perform tomotherapy in the treatment of cervical cancer intensity-modulated radiotherapy is that the dose homogeneity, dose conformity, and dose distribution of planned target volume (PTV) [11-15]. The mean peak dose of the phacoemulsification beam generated during the acquisition of the MVCT image of tomotherapy was $0.336 \mathrm{mGy}$ in the coarse mode and $0.73 \mathrm{mGy}$ in the fine 
mode at 1 time. The mean dose was $10.089 \mathrm{mGy}$ and up to $22.872 \mathrm{mGy}$. The mean value of the intensity-controlled radiotherapy using tomotherapy was $895.83 \mathrm{mGy}$. This result implies that the maximum radiation dose that the patient receives during the entire treatment process is 0.91 Gy when combined with MVCT, which is an imageguiding process, and intensity-modulated radiation therapy. This means that an approximate dose of $1 \mathrm{~Gy}$ can reach the patient and damage the lens. As mentioned in the introduction, the lens can exhibit lens opacity with a threshold dose of 0.5-2 Gy for radiation, as well as cataracts at $4 \mathrm{~Gy}$. In conclusion, it is necessary to design a computerized treatment plan that considers radiationsensitive organs, such as the lens, in the treatment of head and neck cancer with intensity-modulated radiation therapy. It is necessary to carefully recognize doses other than the treatment dose and reduce secondary side effects.

\section{Conclusion}

In the image induction process, the minimum dose should be given considering the location and size of the tumor and adjacent organs. There are various sites and sizes of nasopharynx, oropharynx, and oral cavity in the head and neck of patients with head and neck cancer. We hope that further studies will be conducted on salivary glands, which are important when treating head and neck cancer, as well as the lens and the basic data.

\section{Acknowledgement}

Jae-Won Choi and Hae-Kag Lee equally contributed to this work. They are co-authors of first.

This work was supported in part by the Soonchunhyang University Research Fund.

\section{References}

[1] J. Bak, K. Jeong, K. C. Keum, and S. W. Park, J. Korean Soc. Ther. Radiol. Oncol. 24, 294 (2006).

[2] S. R. Baek, S. Y. Chae, H. O. Kim, S. W. Lee, S. J. Oh, K. C. Im, D. H. Moon, J. S. Kim, and J. S. Ryu, Nucl. Med. Mol. Imaging. 43, 535 (2009).

[3] B. V. Worgul, G. R. Merriam, A. Szechter, and B. D. Srinivasan, Arch Ophthalmol. 94, 999 (1976).

[4] T. Pawlicki, G. Luxton, Q. T. Le, D. Findley, and C. M. Ma, Int. J. Radiat. Oncol. Biol. Phys. 59, 293 (2004).

[5] M. W. Kan, L. H. Leung, W. Wong, and N. Lam, Int. J. Radiat. Oncol. Biol. Phys. 70, 272 (2008).

[6] H. Mok, C. H. Crane, M. B. Palmer, T. M. Briere, S. Beddar, M. E. Delclos, S. Krishnan, and P. Das, Radiat. Oncol. 29, 586 (2011).

[7] J. Burmeister, N. Alvarado, S. Way, P. McDermott, T. Bossenberger, H. Jaenisch, R. Patel, and T. Washington, Med. Dosim. 33, 6 (2008).

[8] D. Baycan, D. Karacetin, A. Y. Balkanay, and Y. Barut, Jpn. J. Radiol. 30, 819 (2012).

[9] M. W. Mcdonald, K. D. Godette, E. K. Butker, L. W. Davis, and P. A. S. Johnstone, Int. J. Radiat. Oncol. Biol. Phys. 72, 1031 (2008).

[10] J. J. Nuyttens, J. M. Robertson, D. Yan, and A. Martinez, Int. J. Radiat. Oncol. Biol. Phys. 53, 497 (2002).

[11] Y. J. Won, J. H. Cho, and S. C. Kim, J. Magn. 21, 133 (2016).

[12] C. W. Choi, J. W. Hong, C. S. Park, and J. O. Ahn, J. Magn. 21, 293 (2016).

[13] S. S. Yom, Z. Liao, H. H. Liu, S. L. Tucker, C. S. Hu, X. Wei, X. Wang, S. Wang, R. Mohan, J. D. Cox, and R. Komaki, Int. J. Radiat. Oncol. Biol. Phys. 68, 94 (2007).

[14] T. Song, Y. Li, and L. Zhou, Med. Phys. 43, 3541 (2016).

[15] A. D. Yock, A. S. Garden, L. E. Court, B. M. Beadle, L. Zhang, and L. Dong, Int. J. Radiat. Oncol. Biol. Phys. 87, 596 (2013). 